\title{
Influence of Drug Use on Morbidity and Mortality in Heatstroke
}

\author{
Michael Levine • Frank LoVecchio • \\ Anne-Michelle Ruha • Gregory Chu • Pedro Roque
}

Published online: 24 March 2012

(C) American College of Medical Toxicology 2012

\begin{abstract}
Numerous medications and illicit drugs can predispose an individual to heat illness, primarily by altering thermoregulation by either increasing endogenous heat production or impairing heat dissipation. This study sought to determine if use of such drugs was associated with more severe illness in patients presenting with heatstroke. A case control study was conducted on adult patients (age, $\geq 14$ years) admitted to an intensive care unit with an admitting diagnosis of heatstroke at two academic teaching hospitals in Phoenix, AZ, between 31 August 2005 through 31 July 2010. Subjects were classified as "users" if they admitted to taking a drug on a pre-defined list of drugs associated with abnormal thermal homeostasis, or if a urine test for drugs of abuse revealed the presence of an amphetamine or cocaine. Similarly, subjects who did not take such drugs were considered "non-users." Seventy-eight patients were identified, with complete medication histories available for 74 of 78 subjects. The overall prevalence of drug utilization was $41.9 \%$ (31 of 74). The
\end{abstract}

M. Levine $(\varangle) \cdot$ F. LoVecchio $\cdot$ A.-M. Ruha

Department of Medical Toxicology,

Banner Good Samaritan Medical Center,

Phoenix, AZ, USA

e-mail: Michael.Levine@bannerhealth.com

M. Levine

Department of Emergency Medicine,

Section of Medical Toxicology, University of Southern California,

Los Angeles, CA, USA

F. LoVecchio $\cdot$ P. Roque

Department of Emergency Medicine, Maricopa Medical Center, Phoenix, AZ, USA

G. Chu

Department of Critical Care Medicine,

Banner Good Samaritan Medical Center,

Phoenix, AZ, USA median length of stay was 3.0 days for the non-users compared with 9.0 days for "users." There was no difference between users and non-users with regard to mortality. Drugs that impair thermoregulation are frequently encountered in patients admitted for heatstroke. Patients taking such drugs may experience increased morbidity over those patients not taking such drugs.

Keywords Heatstroke $\cdot$ Heat illness $\cdot$ Drugs $\cdot$ Heat . Medications

\section{Introduction}

The term heat illness comprises numerous clinical entities which exist along a spectrum. Heat cramps are on the milder end of the spectrum, with heat exhaustion existing in the middle of the spectrum, and heatstroke comprising the most serious form of heat-related illness [1]. Traditionally, heatstroke is defined as the presence of a core body temperature greater than $40^{\circ} \mathrm{C}$ along with neurologic abnormalities (e.g., seizure, encephalopathy, etc.) [2]. Complications including rhabdomyolysis, renal failure, hepatocellular injury, and disseminated intravascular coagulation are common and contribute to the high mortality associated with this condition $[2,3]$.

Numerous factors, including extremes of age, concurrent cardiovascular disease or mental illness, poor level of physical fitness, and performance of strenuous outdoor activities, have been previously identified as potential risk factors for the development of heat-related illness [4]. In addition, it has been suggested that drugs that impair heat dissipation and those which increase endogenous heat production may further predispose to heat-related illness [5-8]. However, the magnitude of this association has not been previously investigated. 
Various medications with antimuscarinic properties, including many of the neuroleptic agents, diphenhydramine, benztropine, and others impair heat dissipation by preventing sweating. Other agents, such as cocaine or amphetamine, can increase endogenous heat production. The primary purpose of this study is to determine if patients using such drugs who are admitted for heatstroke experience greater morbidity or mortality compared with those patients not using such drugs. A secondary purpose of the study is to determine the overall prevalence of drug utilization among patients admitted with heatstroke.

\section{Methods}

This study was a case-control study performed at two urban, academic medical centers in Phoenix, AZ. Subjects who were admitted to an intensive care unit between 31 August 2005 through 31 July 2010 were identified via a search of ICD-9 diagnoses. The study received approval by the institutional review board at each of the participating sites.

Study Subjects The records of all patients with an admitting diagnosis of "heatstroke and sunstroke" (ICD-9 code 992.0); "effects of heat and light" (ICD-9 code 992); "heat exhaustion, anhydrotic" (ICD-9 code 992.3); "unspecified heat" (ICD-9 code 992.9); and "other specified heat effects" (ICD-9 992.8) were screened. Of these subjects, those who had a heat-related illness other than heatstroke (e.g., heat exhaustion, heat cramps, heat syncope, dehydration) were excluded. Thus, the final study population was comprised of adult patients (at least 14 years of age) with an admitting diagnosis of heatstroke.

Subjects in whom bacteremia was present on initial blood cultures were excluded. Patients who either did not have blood cultures or who had initial negative cultures and subsequently developed positive blood cultures during their stay were included as bacteremia in those cases was felt to likely be a complication, rather than the initial cause, of the illness. In addition, if a patient had multiple visits during the study period, only the first hospitalization was included.

Patients were classified as "users" if their medication history included a drug which was found on a list of medications that would predispose to heat illness. This list, which includes medications which increase heat production, impairs heat dissipation, and diuretics were created a priori (Table 1). In addition, patients who were known by history or urine drug screen to have ingested cocaine or an amphetamine were also considered "users." Subjects not known to be consuming medications that impair heat dissipation (Table 1) and with negative urine drug screens (if performed) were classified as "non-users."
Table 1 Drugs which may predispose to heat illness

All typical or atypical antipsychotics

All tricyclic antidepressants

Atropine

Benztropine

Cyclobenzaprine

Diphenhydramine

Hydrochlorothiazide

Furosemide

Metaclopramide

Methylphenidate

Oxybutynin

Prochlorperazine

Promethazine

Scopolamine

Spironolactone

Stimulants (amphetamine, amphetamine derivatives, cocaine)

Outcome Parameters The primary outcome parameter was morbidity or mortality during the index hospitalization of "users" compared to "non-users." Increased length of stay was used as a surrogate marker for increased morbidity. A secondary outcome measure was to determine the overall prevalence of drug utilization in this population.

Data Collection Data were abstracted onto a pre-designed data abstraction form and subsequently entered into a spreadsheet by one investigator at each study site. Before performing data abstraction, each reviewer received a standardized training in systematic chart review. Ten percent of the charts were randomly reviewed by a second investigator to double check accuracy of abstraction. A kappa statistic was performed to assess interrater reliability.

The data abstracted from the medical records included demographic information (age and sex), laboratory studies (initial and worst renal function, hepatic function including prothrombin time, creatinine kinase, urine drug screen results), and vital sign parameters. In addition, a medication history was determined for each patient by review of the physician and nursing documentation from both the emergency department and in-patient records. The date of visit was also recorded, and subsequently, the ambient temperature in downtown Phoenix was determined for the day of admission based on review of the Old Farmer's Almanac.

Data Analysis Independent associations were assessed via chi-squared test and Fisher exact test (as appropriate) for categorical variables. Means and interquartile ranges were used to assess ordinal data that were normally distributed. As some of the data were not normally distributed based on the chi-square goodness-of-fit test, nonparametric analysis was performed via the Mann-Whitney $U$ test. Odds ratios 
with $95 \%$ confidence intervals were calculated to evaluate associations between unique variables and length of stay.

The following variables were compared versus length of stay and outcome: need for mechanical ventilation, need for hemodialysis, use of cocaine, use of methamphetamine, use of pre-defined medications, use of any xenobiotic (cocaine, amphetamine, or any pre-defined medication), initial and lowest $\mathrm{pH}$, platelets, hemoglobin, and the initial and maximal lactic acid, creatinine kinase, troponin, prothrombin time, creatinine, aspartate aminotransferase (AST), alanine aminotransferase, and white blood cell count. To control the desired outcome for multiple comparisons, binary logistic regression was subsequently performed. All statistical analyses were performed using STATA ${ }^{\text {TM }} 2007$ College Station, TX.

\section{Results}

During the study period, a total of 574 patients were identified, of which 78 patients met study inclusion criteria. The mean ( \pm standard deviation) age was 49.6 years $( \pm 19.2)$. Nine $(11 \%)$ subjects were women. Nine (11\%) subjects died, and an additional 15 of $78(19 \%)$ required rehabilitation or skilled nursing care upon discharge.

The mean daily ambient temperature was $41.7^{\circ} \mathrm{C}( \pm 8.02)$, while the mean core body temperature was $40.8^{\circ} \mathrm{C}( \pm 1.35)$. There was no association between ambient outdoor temperature and mortality or length of stay $\left(r^{2}=0.26,-0.1\right.$ to 0.56$)$.

Medication histories were not available for 4 of 78 (5\%) subjects. Of the 78 subjects, 43 (55\%) were classified as "non-users," while 31 (40\%) used at least one of the predefined drugs and were classified as "users." Thus, among patients with known medication histories, the overall prevalence of recreational and pharmaceutical drug usage was $41.9 \%$ (31 of 74). The median length of stay was 3.0 days for non-users and 9.0 days for users $(p=0.005$; $95 \%$ CI $1-$ $3.5)$ and was not affected by advanced age $(r=0.94$, $p=0.41)$. Table 2 lists the aggregate number of agents identified among the users.

Urine drug screens were performed on 61 of 78 (78.2 \%) patients. Among those patients who had a urine drug screen performed, the cocaine screen was positive in seven patients while the amphetamine screen was positive in 12 patients, including two who also had a positive cocaine screen. Thus, 17 of $61(27.8 \%)$ patients in whom a urine drug screen was performed were positive for cocaine or amphetamines. Among those patients whose urine drug screens were negative, 11 of $44(25 \%)$ were classified as "users" based on medication histories. Of the 17 patients in whom no urine drug screen was performed, 3 of 17 (17.6\%) were classified as "users" based on the medication history. No patient who had a negative urine drug screen had admitted to using
Table 2 Number of times each agent was identified among the study population

\begin{tabular}{lc}
\hline Agents identified & $\begin{array}{c}\text { Number of times the agent } \\
\text { was identified }\end{array}$ \\
\hline Amphetamine & 10 \\
Antipsychotic & 8 \\
Benztropine & 2 \\
Cocaine & 7 \\
Cyclobenzaprine & 1 \\
Diphenhydramine & 2 \\
Diuretic & 3 \\
Tricyclic antidepressant screen & 4 \\
\hline
\end{tabular}

cocaine or amphetamines. The urine drug screens performed included both immunoassay screens and comprehensive urine drug screens (gas chromatography-mass spectrometry). Which screen was performed was a function of which of the two hospitals the patient presented.

The data were subsequently analyzed using binary logistic regression to correct for confounding variables. The variables maximal temperature, maximal heart rate, maximal creatinine kinase, initial creatinine, initial white blood count, need for mechanical ventilation, and use of amphetamines were associated with increased length of stay. These significant variables were then controlled for multiple comparisons with logistic regression and Bonferroni correction where indicated. Following control for multiple comparisons, only heart rate (OR 1.007, $p=0.007)$ and mechanical ventilation (OR 1.31, $p=0.047)$ remained statistically significant for predicting increase in length of stay. Users required mechanical ventilation more frequently than nonusers ( 68 vs. $29 \%, p=0.0018$ ). In addition, users experienced many other complications compared to non-users, including higher maximal creatinine kinases, a higher degree of transaminitis, and more renal failure. These results are presented in Table 3.

Nine subjects (11\%) died. Of these, two were non-users, and four were users. The remaining three patients either presented in cardiac arrest or died before further history could be obtained.

Table 3 Median and interquartile ranges of various complications comparing users and non-users

\begin{tabular}{lccc}
\hline & Users & Non-users & $p$ value \\
\hline Maximal CK & $2,914(595-14,154)$ & $420(132-4,899)$ & 0.0043 \\
Maximal AST & $630(145-3,330)$ & $81(41-359)$ & 0.0003 \\
$\begin{array}{l}\text { Maximal } \\
\text { creatinine }\end{array}$ & $2(1.4-3.2)$ & $1.3(1.1-1.8)$ & $<0.001$ \\
\hline
\end{tabular}

$C K$ creatinine kinase, $A S T$ aspartate aminotransferase 
Using binary logistic regression, no variable remained associated with increased mortality. Subgroup analysis failed to reveal any significant difference between those who used illegal drugs (cocaine or amphetamines) and those who used prescribed xenobiotics. A kappa statistic was calculated and was 0.85 .

\section{Discussion}

A wide variety of prescription medications and illicit substances may predispose to heatstroke. Nearly half of the patients in our study population used drugs known to alter thermoregulation. This subgroup of patients had a median length of hospital stay that was three times longer than the patients without similar drug use. Despite this longer length of stay, we did not find an increase in mortality.

In the general population, the prevalence of use of drugs that predispose to heat illness is unknown but is likely much lower than the rate of $42 \%$ discovered in our study cohort. Most other studies of patients with heatstroke have not looked specifically at use of thermoregulation-altering drugs [9-11], although one small case series of patients that presented to an ED during a heat wave reported 6 of $8(75 \%)$ patients had taken drugs that could contribute to hyperthermia [12].

The drugs most commonly encountered in our study population included sympathomimetics (e.g., cocaine or amphetamines), anticholinergics (e.g., benztropine, diphenhydramine, antipsychotics), and diuretics. Both cocaine and amphetamines are sympathomimetic agents, which can cause hyperthermia due to an increase in endogenous heat production [13]. The risk of sympathomimetic-induced hyperthermia is increased with high ambient temperatures [14-16]. Marzuk and colleagues [14] examined the effect of ambient temperature on cocaine-related deaths. They concluded that cocaine-related deaths increased on hot days. However, the authors did not account for possible confounders, thereby limiting the results of the study [17]. It is not known if patients were taking other medications that may alter thermoregulation. Amphetamines such as methylenedioxymethamphetamine (ecstasy), can also cause hyperthermia as part of a hyperserotonergic state [13]. It should be noted that no patient in the current study was diagnosed with serotonin syndrome.

Numerous agents have antimuscarinic properties, including the tricyclic antidepressants, most antipsychotics, benztropine, cyclobenzaprine, and antihistamines such as diphenhydramine. These agents are known to impair heat dissipation [18]. As a result, these agents can predispose to hyperthermia.

Diuretics have also been associated with heatstroke [19]. It is not clear if the diuretics are creating a state of dehydration, which may predispose to heat illness. Alternatively, the diuretics may simply be a marker of cardiovascular disease, which is known to be a risk factor for the development of non-exertional heatstroke [19].

We attempted to identify other predictors for increased length of stay in our patients with drug use as compared to those without drug use. Heart rate and respiratory failure were the only variables that were found to be statistically significant, but we believe these are both markers for severe illness rather than independent risk factors. While statistically significant, the small difference in heart rate is unlikely to be clinically relevant. Users had a much greater need for mechanical ventilation than non-users. The difference in this variable likely represents an increased severity among the users compared with the non-users and may account for much of the difference in length of stay. Due to the retrospective nature, however, it is impossible to state whether increased morbidity accounted for the full difference of length of stay observed.

It is well established that patients drinking alcoholic beverages, those ingesting certain illicit drugs (e.g., cocaine or amphetamines), and those participating in vigorous, strenuous outdoor activities in hot weather are at risk for heat-related illness $[2,20,21]$. It is also established that substance abuse commonly co-exists among patients with mental illness [22, 23]. It is possible that poor judgment due to underlying psychiatric illness prevents many patients from moving indoors or stopping vigorous activity during the early stages of heat illness. If this is the case, it may be that the underlying mental illness is the risk factor, and the drug use is simply a marker of the underlying mental illness. One study looking at characteristics of patients who died in Chicago during the 1995 heat wave found an increased odds of death (OR 3.5; $95 \%$ 1.7-7.3) associated with "mental problems" [24]. However, because a complete medication history was not provided for subjects, it is not possible to determine from their manuscript if subjects with mental illness were taking medications or illicit drugs that are known to affect thermoregulation.

This study has several limitations. First, the study is retrospective. As a result, the conclusions are limited by the quality and completeness of data recorded in the medical record. To minimize these effects, the primary data abstracted were limited to continuous variables (e.g., recorded heart rate, creatinine, length of stay, etc.) or categorical variables which were subject to little interpretation bias (e.g., presence or absence of a pre-defined medication on the medication history). Choosing these specific variables to be abstracted likely reduced, if not eliminated, abstractor bias and thus minimized some of the limitations of the retrospective review [25].

It is possible that some patients had incomplete or inaccurate drug histories obtained. Because serum drug levels were not routinely obtained, it is not possible to assess 
compliance. Thus, it is possible that some patients were classified as "users" based on their reported history, but were in fact non-users if they were not compliant with their medications.

Furthermore, not every patient in this study had a urine drug screen performed. Certainly, some patients may not be forthcoming about their drug use. Given the high prevalence of both cocaine and amphetamine in this population, it is unlikely that underreporting and underdetecting contributed significantly to the findings. Nonetheless, it is possible that some patients may have been classified as "non-users" who actually did have illicit drug use that either was not detected or not reported. However, even if underreporting or underdetection were to occur, one would hypothesize the observed differences to be larger. Thus, if any "user" was classified as a "non-user" based on the lack of a urine drug screen, we would expect the true differences between the two groups to be even greater.

This study examined patients who presented to the emergency department and did not include data from the medical examiner's office. Thus, the most severe patients (e.g., those who died before presenting to the hospital) would have not been included in the study. This scenario may explain the lack of observed mortality difference. Thus, extrapolating the results of this study to all patients with heatstroke may be limited, as those patients who died before presentation to a hospital were not included.

The use of a urine drug screen does raise some limitations. The presence of a drug on a drug screen may imply utilization, but does not imply acute intoxication [26]. In general, the cocaine or amphetamine test will remain positive for 2 to 3 days after last use. The screen for cocaine relies on the detection of the metabolite benzoylecognine. The cocaine screen is generally considered to be fairly accurate with few false positives. In contrast, the amphetamine screen is associated with numerous false positives, including phenylpropanolamine, brompheniramine, bupropion, trazadone, chlorpromazine, promethazine, and ranitidine [27]. No patient in our study was known to be taking ranitidine. While many agents can cause false-positive amphetamine screens, many of these agents would be expected to result in impaired heat dissipation (chlorpromazine, promethazine) or increase endogenous heat production (brompheniramine, phenylpropanolamine). Thus, even if the positive amphetamine screen represented a false positive, it is unlikely that the false-positive screen would substantially change the results. Furthermore, it is possible that some patients had a drug metabolite detected without having pharmacologic active drug. If this were the case, it could have overemphasized our findings.

Hyperthyroidism can cause hyperthermia as part of thyroid storm. No patient had a diagnosis of thyroid storm or thyrotoxicosis as a discharge diagnosis. Because the data were not obtained as part of a prospective protocol, thyroid studies were not obtained in every patient. Thus, it is possible, although unlikely, that thyroid disease was misdiagnosed as heatstroke.

Our inferential statistical analysis is underpowered to show any direct link between drug use and increased death in patients with hyperthermia.

It has been suggested that public health officials identify high-risk populations prior to heat waves in order to prevent heat-related illness and death. While patients at the extremes of age and those with underlying medical or psychiatric illness are traditionally considered to be high-risk populations, based on this study, those individuals using certain drugs may also be considered high risk. While it remains a possibility that drug use simply serves as a marker for underlying medical or psychiatric illness, it would be prudent to consider patients taking medications that impair thermoregulation to be in a potentially high-risk group and plan accordingly during heat waves.

\section{Conclusion}

The use of medications that impair heat dissipation or increase endogenous heat production is commonly encountered among patients admitted to an intensive care unit with heat-related illness. Furthermore, the use of such medications may be associated with more severe illness, but is not associated with increased mortality.

\section{References}

1. Lugo-Amador NM, Rothenhaus T, Moyer P (2004) Heat-related illness. Emerg Med Clin North Am 22:315-327

2. Bouchama A, Knochel JP (2002) Heat stroke. N Engl J Med 346:1978-1988

3. Waters TA (2001) Heat illness: tips for recognition and treatment. Cleve Clin J Med 68:685-687

4. Centers for Disease Control and Prevention (CDC) (2010) Heat illness among high school athletes: United States, 2005-2009. MMWR Morb Mortal Wkly Rep 59:1009-1013

5. Centers for Disease Control and Prevention (CDC) (2005) Heatrelated mortality: Arizona, 1993-2002 and United States, 19792002. MMWR Morb Mortal Wkly Rep 54:628-630

6. Becker JA, Stewart LK (2011) Heat-related illness. Am Fam Physician 83:1325-1330

7. Kwok JS, Chan TY (2005) Recurrent heat-related illnesses during antipsychotic treatment. Ann Pharmacother 39:1940-1942

8. Khosla R, Guntupalli KK (1999) Heat-related illness. Crit Care Clin 15:251-263

9. Carter R 3rd, Cheuvront SN, Williams JO et al (2005) Epidemiology of hospitalizations and deaths from heat illness in soldiers. Med Sci Sports Exerc 37:1338-1344

10. Zeller L, Novack V, Barski L et al (2011) Exertional heatstroke: clinical characteristics, diagnostic and therapeutic considerations. Eur J Intern Med 22:296-299

11. Casa DJ, Armstrong LE, Ganio MS et al (2005) Exertional heat stroke in competitive athletes. Curr Sports Med Rep 4:309-317 
12. Martinez M, Devenport L, Saussy J et al (2002) Drug-associated heat stroke. South Med J 95:799-802

13. Devlin RJ, Henry JA (2008) Clinical review: major consequences of illicit drug consumption. Crit Care 12:202

14. Marzuk PM, Tardiff K, Leon AC et al (1998) Ambient temperature and mortality from unintentional cocaine overdose. JAMA 279:1795-1800

15. Green AR, O'Shea E, Saadat KS et al (2005) Studies on the effect of MDMA ('ecstasy') on the body temperature of rats housed at different ambient room temperatures. Br J Pharmacol 146:306-312

16. Green AR, O'Shea E, Colado MI (2004) A review of the mechanisms involved in the acute MDMA (ectasy)-induced hyperthermic response. Eur J Pharmacol 500:3-13

17. Kilbourne EM (1998) Cocaine use and death during heat waves. JAMA 279:1828-1829

18. McAllen KJ, Schwartz DR (2010) Adverse drug reactions resulting in hyperthermia in the intensive care unit. Crit Care Med 38(6 Suppl): S244-S252

19. Hausfater P, Megarbane B, Dautheville S et al (2010) Prognostic factors in non-exertional heatstroke. Intensive Care Med 36:272-280

20. Bytomski JR, Squire DL (2003) Heat illness in children. Curr Sports Med Rep 2:320-324
21. Centers for Disease Control and Prevention (CDC) (1996) Extreme heat. US Department of Health and Human Services, CDC, Atlanta, GA. Available at: http://emergency.cdc.gov/disasters/extremeheat/. Accessed 19 Mar 2012

22. Green AI, Tohen MF, Hamer RM (2004) First episode schizophreniarelated psychosis and substance use disorders: acute response to olanzapine and haloperidol. Schizophr Res 66:125-135

23. Substance Abuse and Mental Health Services Administration (2010) Results from the 2009 National Survey on Drug Use and Health: Mental Health Findings (Office of Applied Studies, NSDUH series h39, HHS Publication No SMA 10-4609). Rockville, MD

24. Semenza JC, Rubin CH, Falter KH et al (1996) Heat-related deaths during the July 1995 heat wave in Chicago. N Engl J Med 335:84-90

25. Gilbert EH, Lowenstein SR, Koziol-McLain J et al (1996) Chart reviews in emergency medicine research: what are the methods? Ann Emerg Med 27:305-308

26. Levine M, Brooks DE, Truitt CA et al (2011) Toxicology in the ICU: Part 1: General overview and approach to treatment. Chest 140:795-806

27. Brahm NC, Yeager LL, Fox MD et al (2010) Commonly prescribed medications and potential false-positive urine drug screens. Am J Health Syst Pharm 67:1344-1350 